\title{
Case Report \\ The Challenges of Dialysis in Systemic Sclerosis: Between the Devil and the Deep Blue Sea?
}

\author{
N. Brown, ${ }^{1}$ A. Summers, ${ }^{1}$ M. C. Venning, ${ }^{1}$ and I. N. Bruce ${ }^{2}$ \\ ${ }^{1}$ Renal Unit, Manchester Royal Infirmary, Central Manchester Foundation Trust, Oxford Road, Manchester M13 9WL, UK \\ ${ }^{2}$ Arthritis Research Unit, University of Manchester, Oxford Road, Manchester M13 9PL, UK
}

Correspondence should be addressed to N. Brown, nina.brown@cmft.nhs.uk

Received 9 July 2012; Accepted 26 August 2012

Academic Editors: S. Kavukcu, K. C. Siamopoulos, and W. Sulowicz

Copyright () 2012 N. Brown et al. This is an open access article distributed under the Creative Commons Attribution License, which permits unrestricted use, distribution, and reproduction in any medium, provided the original work is properly cited.

We present the case of a patient with systemic sclerosis (SSc) and end stage renal disease (ESRD) who experienced complications of both peritoneal and haemodialysis. We review previously reported outcomes of patients with systemic sclerosis on dialysis and discuss potential shared mechanisms in both the disease pathogenesis and dialysis-related complications, particularly with regards to encapsulating peritoneal sclerosis (EPS).

\section{Introduction}

Systemic sclerosis (SSc) is a rare but important cause of end-stage renal disease (ESRD). We present a case which outlines dialysis complications which may potentially arise as a consequence of the underlying disease. This case highlights the challenges which may be faced by the patient and clinician in choosing the optimum modality of renal replacement therapy (RRT) for those with systemic sclerosis.

\section{Case Report}

We describe the case of a 51-year-old female who presented early in 2003 with a polyarthritis. She was initially thought to have rheumatoid arthritis and was treated with intramuscular triamcinolone and hydroxychloroquine. This was subsequently discontinued due to side effects of vomiting and diarrhoea. She presented shortly following this to a district General Hospital with renal failure, malignant hypertension, and microangiopathy. During her acute admission, she developed pulmonary oedema and seizures requiring intubation, intensive care admission, and haemofiltration. Renal biopsy was contraindicated at that time due to low platelets and bleeding risk. Due to nonrecovery of renal function, she was transferred to our renal unit for Tenckhoff catheter insertion and commenced automated peritoneal dialysis.
During this admission, she was reviewed by the Rheumatology team who noted a history of Raynaud's phenomenon as well as the polyarthropathy and commented on some tight skin over the distal phalanges. Her immunological tests demonstrated a positive antinuclear antibody, titre $1: 300$, with both a speckled and nucleolar pattern. However, double-stranded DNA antibody was negative, and complement was normal. In view of her clinical and immunological findings, it was felt that her microangiopathy was related to an underlying connective tissue disorder, potentially a lupus/systemic sclerosis overlap. She started on low-dose prednisolone to which her musculoskeletal symptoms responded and was subsequently maintained on $5 \mathrm{mg}$ prednisolone a day. Following the acute presentation, she started on an angiotensin converting enzyme inhibitor and blood pressure came under control.

Under followup over the next 2 years she developed more pronounced sclerodactyly and gastric antral vascular Ectasia (or watermelon stomach) with these clinical features confirming her diagnosis of systemic sclerosis. She became transiently positive for lupus anticoagulant in 2005, but this was negative on repeat testing along with negative anticardiolipin antibodies and no thrombotic events. She remained fairly stable, though dialysis dependent, for 4 years with the only additional treatment required for her connective tissue disorder being annual epoprostenol infusions for her 


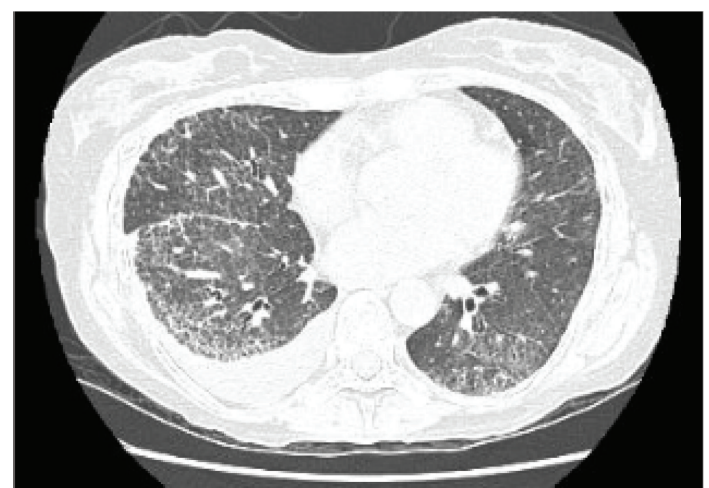

FIgURE 1: HRCT showing UIP (followup scan, May 2008).

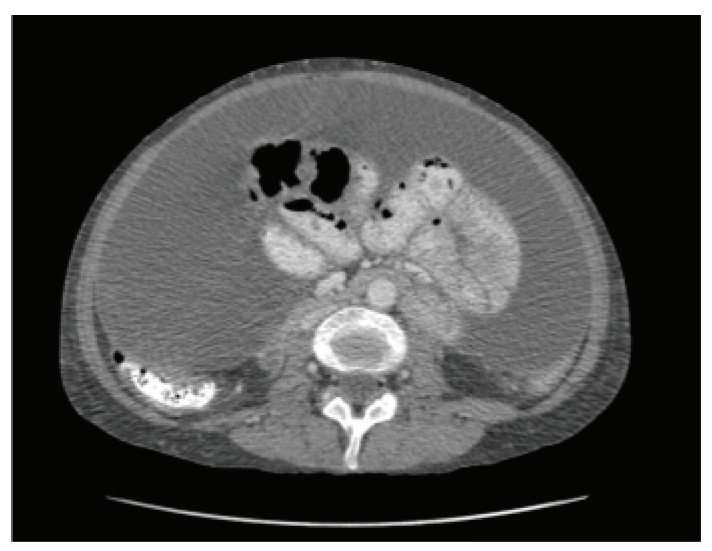

FIGURE 2: CT abdomen showing ascites and thickened peritoneum (June 2010).

A further attempt at arteriovenous fistula formation was unsuccessful, and dialysis was continued via a catheter. In spite of treatment with radiotherapy and tamoxifen her cancer progressed with multiple liver, splenic, and bone metastases presented April 2011. Following commencement of chemotherapy in May 2011, she was admitted with neutropenia and pneumonia and died shortly following admission.

We present this case to highlight the difficulties faced when choosing a dialysis modality for patients with SSc due to the potential impact of this disease upon the success of dialytic therapies.

\section{Discussion}

We present a woman with connective tissue disease and a seemingly progressive systemic sclerotic phenotype. This was characterised by Raynaud's phenomenon, sclerodactyly, and facial telangectasia, with late development of watermelon stomach and pulmonary fibrosis.

It has long been recognized that the most serious complication of peritoneal dialysis is the development of encapsulating peritoneal sclerosis (EPS). It is characterised by a progressive, intra-abdominal, inflammatory process resulting in sheets of fibrous tissue which cover, bind, and constrict the viscera, thereby compromising the motility and function of the bowel. At the molecular level, there are many common fibrotic and angiogenic factors (TGF, VEGF) which have been implicated in the pathogenesis of systemic sclerosis and EPS $[1,2]$. It is therefore possible that patients with systemic sclerosis are more likely to develop multiorgan fibrosis, including the spectrum of EPS. However, to our knowledge, this is the first report of any possible association between EPS and systemic sclerosis. Moreover, the question of technique survival in PD patients with this sclerotic phenotype still needs investigation.

A recent publication reviewed dialysis outcomes of 127 SSc patients in the Australia/New Zealand (ANZDATA) dialysis registry [3]. $50 \%$ of these patients were treated with $\mathrm{PD}$ reflecting the much higher use of this dialysis modality The surgical intervention successfully alleviated the bowe obstruction, and no postoperative complications occurred. 
in these countries. Incidence of EPS was not specifically reported in this population. Cause of death was primarily from cardiovascular disease followed by withdrawal from dialysis, which may indicate peritoneal dialysis failure. Median survival on dialysis in this population was only 2.43 years, perhaps not providing long enough exposure to peritoneal dialysis to develop the complication of EPS.

This case of a patient with systemic sclerosis and EPS, yet without any of the conventional risk factors (e.g., recurrent peritonitis, excessive $\mathrm{PD}$ vintage), raises the question as to whether systemic sclerosis itself may predispose to EPS. Similarly, the failure of two fistula attempts is likely to represent a diminished fistula success chance in patients with the vasculopathy of SSc, possibly in part due to circulating profibrotic and angiogenic molecules [4]. It has also been suggested that the presence of antiphospholipid antibodies may play a part in the pathogenesis of vascular events in SSc [5].

Systemic sclerosis patients have impaired flow-mediated vasodilatation, reflecting abnormal endothelial function [6]. There is no data reporting the outcomes of dialysis fistula formation in this population, but events due to atherosclerotic and structural vascular disease are well described in patients with SSc [7].

In summary, further data is required regarding outcomes on dialysis for patients with systemic sclerosis, specifically with regards to vascular access outcomes, peritoneal membrane failure, and development of EPS. This will allow both clinicians and patients to be better informed when making decisions concerning the optimum mode of dialysis in this population.

\section{Acknowledgments}

Dr. N. Brown and Dr. M. Venning have received research Awards from Roche. Professor Bruce has recieved a consulting and speaker bureau from Roche, GSK and Human Genome Sciences, Astra Zeneca, and UCB. Dr. A. Summers has received speaker fees from Baxter Healthcare.

\section{References}

[1] P. J. Margetts, K. H. Oh, and M. Kolb, "Transforming growth factor- $\beta$ : importance in long-term peritoneal membrane changes," Peritoneal Dialysis International, vol. 25, no. 3, pp. S15-S17, 2005.

[2] R. Sgonc and G. Wick, "Pro- and anti-fibrotic effects of TGFbeta in scleroderma," Rheumatology, vol. 47, supplement, pp. v5-v7, 2008.

[3] B. Siva, C. M. Hawley, S. P. McDonald et al., "End-stage kidney disease due to scleroderma-outcomes in 127 consecutive ANZDATA registry cases," Nephrology, Dialysis, Transplantation, vol. 26, no. 10, pp. 3165-3171.

[4] S. Guiducci, O. Distler, J. H. Distler, and M. Matucci-Cerinic, "Mechanisms of vascular damage in SSc-implications for vascular treatment strategies," Rheumatology, vol. 47, supplement 5, pp. v18-v20, 2008.

[5] I. Marie, F. Jouen, M. F. Hellot, and H. Levesque, "Anticardiolipin and anti- $\beta 2$ glycoprotein I antibodies and lupuslike anticoagulant: prevalence and significance in systemic sclerosis," British Journal of Dermatology, vol. 158, no. 1, pp. 141-144, 2008.

[6] F. Bartoli, J. Blagojevic, M. Bacci et al., "Flow-mediated vasodilation and carotid intima-media thickness in systemic sclerosis," Annals of the New York Academy of Sciences, vol. 1108, pp. 283-290, 2007.

[7] K. Au, M. K. Singh, V. Bodukam et al., "Atherosclerosis in systemic sclerosis: a systematic review and meta-analysis," Arthritis and Rheumatism, vol. 63, no. 7, pp. 2078-2090, 2011. 


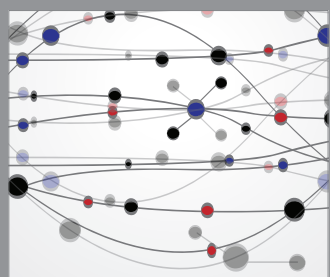

The Scientific World Journal
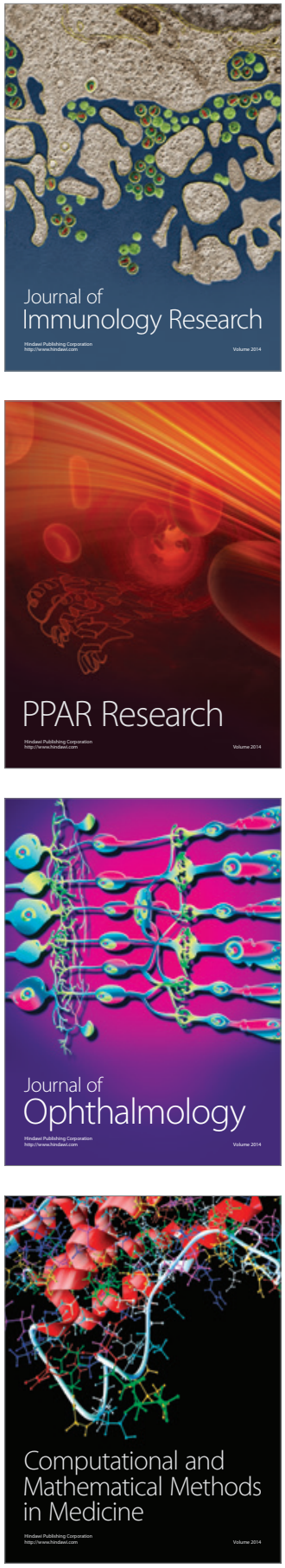

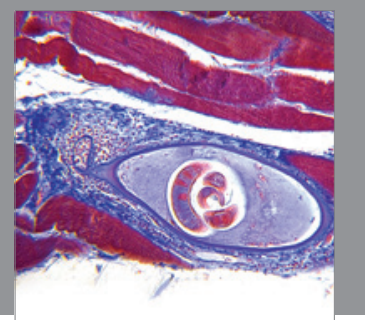

Gastroenterology

Research and Practice
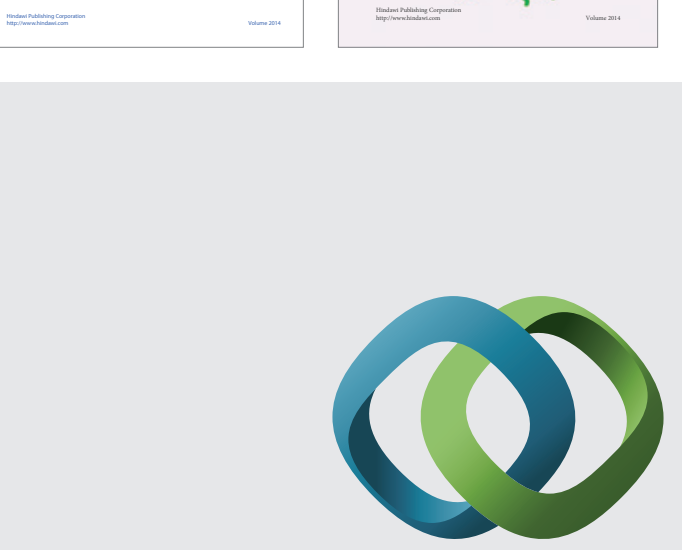

\section{Hindawi}

Submit your manuscripts at

http://www.hindawi.com
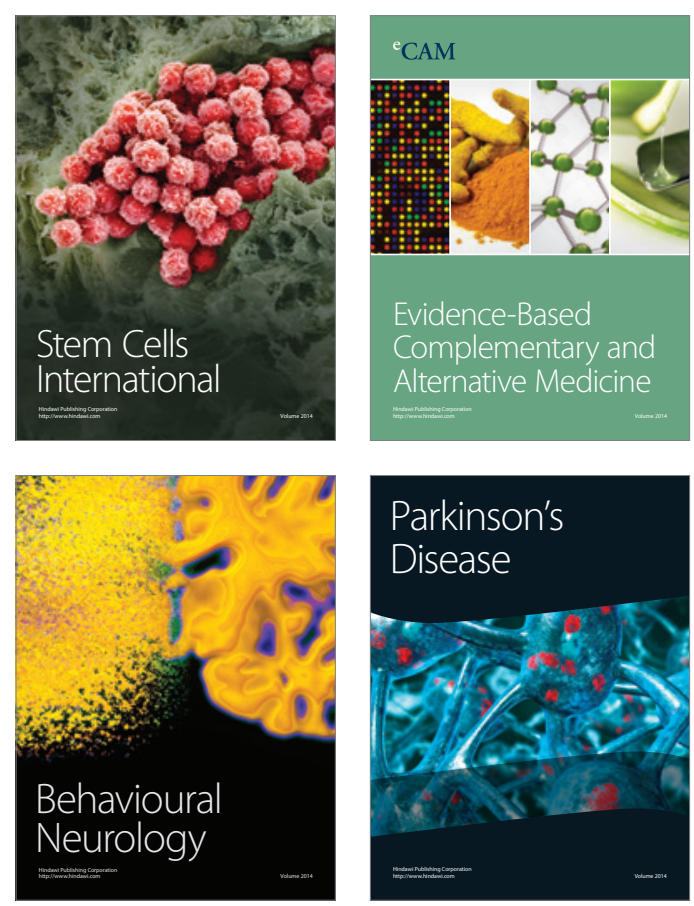

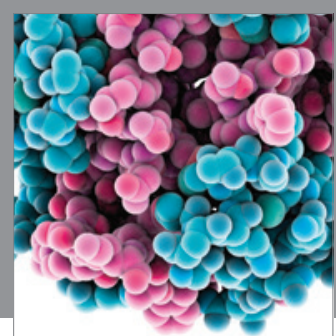

Journal of
Diabetes Research

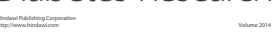

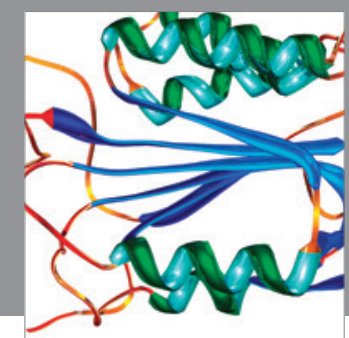

Disease Markers
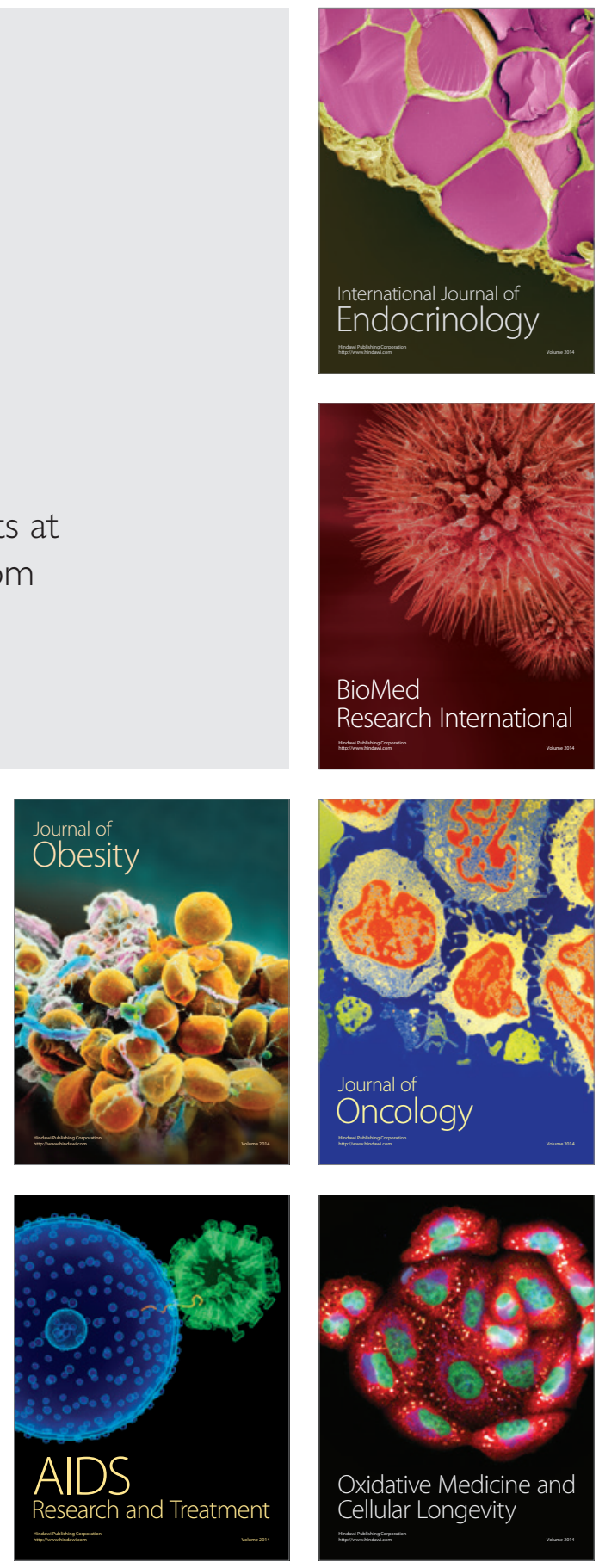